\title{
SHARP ERROR ESTIMATES FOR INTERPOLATORY APPROXIMATION ON CONVEX POLYTOPES
}

\author{
ALLAL GUESSAB* AND GERHARD SCHMEISSER ${ }^{\dagger}$
}

\begin{abstract}
Let $\mathfrak{P}$ be a convex polytope in the $d$-dimensional Euclidean space. We consider an interpolation of a function $f$ at the vertices of $\mathfrak{P}$ and compare it with the interpolation of $f$ and its derivative at a fixed point $y \in \mathfrak{P}$. The two methods may be seen as multivariate analogues of an interpolation by secants and tangents, respectively. For twice continuously differentiable functions, we establish sharp error estimates with respect to a generalized $L^{p}$ norm for $1 \leq p \leq \infty$. The case $p=1$ is of special interest since it provides analogues of the midpoint rule and the trapezoidal rule for approximate integration over the polytope $\mathfrak{P}$. In the case where $\mathfrak{P}$ is a simplex and $p>1$, this investigation covers recent results by S. Waldron [8] and by M. Stämpfle [6].
\end{abstract}

Key words. Interpolation on convex polytopes, sharp error estimates, approximation of functions, approximate integration, approximation of functionals

AMS subject classifications. 41A05, 41A20, 41A44, 41A63, 41A80, 65D05, 65D30

1. Introduction and Notation. Denote by $\mathcal{P}_{1}$ the class of all polynomials in $d$ real variables of degree at most 1 , also called the class of affine functions on $\mathbb{R}^{d}$. Let $\mathfrak{P} \subset \mathbb{R}^{d}$ be a convex polytope of positive measure with vertices $v_{1}, \ldots, v_{n}$, and let $B_{1}, \ldots, B_{n}$ be an associated system of continuous functions on $\mathfrak{P}$ with the following properties:

Non-negativity. For $i=1, \ldots, n$, we have

$$
B_{i}(x) \geq 0 \quad(x \in \mathfrak{P}) .
$$

Linear precision. For every $\lambda \in \mathcal{P}_{1}$, we have

$$
\lambda(x)=\sum_{i=1}^{n} \lambda\left(v_{i}\right) B_{i}(x) .
$$

Warren [10] showed that $B_{1}, \ldots, B_{n}$ can be chosen as rational functions, which are uniquely determined if one requires that each $B_{i}$ have minimal degree. Furthermore, for an arbitrary convex polytope, he presented an algorithm for constructing these functions $B_{1}, \ldots, B_{n}$ in a finite number of steps.

Since vertices of a convex polytope are extremal points, it is easily deduced from the "linear precision" that

$$
B_{i}\left(v_{j}\right)=\delta_{i j} \quad(i, j \in\{1, \ldots, n\}),
$$

where we use Kronecker's delta. As a consequence of (1.2) and (1.3), the functions $B_{1}, \ldots, B_{n}$ are linearly independent and span an $n$-dimensional linear space $\mathcal{R}_{n}$ which contains $\mathcal{P}_{1}$ as a subspace.

By $C(\mathfrak{P}), C^{1}(\mathfrak{P})$, and $C^{2}(\mathfrak{P})$, we denote the spaces of functions which are defined on $\mathfrak{P}$ and are continuous, continuously differentiable, and twice continuously differentiable, respectively.

*Department of Applied Mathematics, University of Pau, 64000 Pau, France, e-mail address: allal.guessab@univ-pau.fr.

${ }^{\dagger}$ Mathematical Institute, University of Erlangen-Nuremberg, 91054 Erlangen, Germany, e-mail address: schmeisser@mi.uni-erlangen.de. 
Next, let $\mathcal{L}$ be a positive linear functional on $C(\mathfrak{P})$. The positivity means that $\mathcal{L}(f)>0$ for every nontrivial non-negative function $f \in C(\mathfrak{P})$.

Examples of such functionals are weighted integrals

$$
\mathcal{L}(f):=\int_{\mathfrak{P}} w(x) f(x) \mathrm{d} x \quad(f \in C(\mathfrak{P})),
$$

where $w$ is integrable and positive on $\mathfrak{P}$ except for a set of measure zero.

For $f \in C(\mathfrak{P})$, we introduce

$$
\|f\|_{p}:=\left(\mathcal{L}\left(|f|^{p}\right)\right)^{1 / p} \quad(1 \leq p<\infty)
$$

and

$$
\|f\|_{\infty}:=\sup _{x \in \mathfrak{P}}|f(x)|
$$

which define norms on $C(\mathfrak{P})$. When $\mathcal{L}$ is given by $(1.4)$ and $w=1$, then $\|\cdot\|_{p}$ is the familiar $L^{p}$ norm. For general $\mathcal{L}$, we may think of $\mathfrak{P}$ as being equipped with a mass distribution such that $\mathcal{L}(1)$ is the total mass of $\mathfrak{P}$. The possibility of having an arbitrary $\mathcal{L}$ is of interest mainly in our applications of the case $p=1$ (see Section 4 ). For this reason, we do not use a weighted supremum norm.

By $\|\cdot\|$, without any subscript, and by $\langle\cdot, \cdot\rangle$, we want to denote the Euclidean norm and the standard inner product in $\mathbb{R}^{d}$.

In this paper, we shall study the linear interpolation operator $\Lambda^{\mathrm{v}}$, defined by

$$
\Lambda^{\mathrm{v}}[f]:=\sum_{i=1}^{n} f\left(v_{i}\right) B_{i} \quad(f \in C(\mathfrak{P})),
$$

which interpolates $f$ at the vertices of $\mathfrak{P}$, and shall compare it with

$$
\Lambda_{y}[f]:=f(y)+D f(y)(\cdot-y) \quad\left(f \in C^{1}(\mathfrak{P})\right),
$$

where $y \in \mathfrak{P}$. Clearly, $\Lambda_{y}[f]$ interpolates $f$ at $y$ and the same holds for the first derivative.

As regards our notation, we want to follow the convention that a superscript in roman type indicates an abbreviation for a word while a subscript in italic type is a mathematical quantity. In particular, the superscript $\mathrm{v}$ shall always refer to interpolation at the vertices. Similarly, we shall use the superscripts sb for smallest ball and $\mathrm{cm}$ for center of mass.

2. Auxiliary Results. For convenient reference, we first state some properties of the operators $\Lambda_{y}$ and $\Lambda^{\mathrm{v}}$ as lemmas.

Lemma 2.1. For $y \in \mathfrak{P}$, the operator $\Lambda_{y}$ has the following properties.

(i) It maps $C^{1}(\mathfrak{P})$ into $\mathcal{P}_{1}$.

(ii) It reproduces functions from $\mathcal{P}_{1}$.

(iii) It approximates convex functions from below.

Proof. The properties (i) and (ii) are obvious. Property (iii) is a well known fact about differentiable, convex functions; see [5, p. 98, Theorem A].

LEMMA 2.2. The operator $\Lambda^{\mathrm{v}}$ has the following properties.

(i) It maps $C(\mathfrak{P})$ into $\mathcal{R}_{n}$.

(ii) It reproduces functions from $\mathcal{R}_{n}$. 
(iii) It approximates convex functions from above.

(iv) If $f, g \in C(\mathfrak{P})$ and $f\left(v_{i}\right) \leq g\left(v_{i}\right)$ for $i=1, \ldots, n$, then $\Lambda^{\mathrm{v}}[f] \leq \Lambda^{\mathrm{v}}[g]$.

Proof. Since $\left\{B_{1}, \ldots, B_{n}\right\}$ is a basis of $\mathcal{R}_{n}$, the properties (i) and (ii) are obvious consequences of the definition of $\Lambda^{\mathrm{v}}$.

Next, it follows from (1.2) that

$$
x=\sum_{i=1}^{n} v_{i} B_{i}(x) \quad(x \in \mathfrak{P}),
$$

which is a representation of $x$ as a convex combination of the vertices of $\mathfrak{P}$. Hence, for a convex function $f$,

$$
f(x)=f\left(\sum_{i=1}^{n} v_{i} B_{i}(x)\right) \leq \sum_{i=1}^{n} f\left(v_{i}\right) B_{i}(x)=\Lambda^{\mathrm{v}}[f](x) \quad(x \in \mathfrak{P}),
$$

and so statement (iii) is verified.

Finally, recalling (1.1), we see that, under the hypothesis of statement (iv),

$$
\Lambda^{\mathrm{v}}[f]=\sum_{i=1}^{n} f\left(v_{i}\right) B_{i} \leq \sum_{i=1}^{n} g\left(v_{i}\right) B_{i}=\Lambda^{\mathrm{v}}[g] .
$$

This completes the proof.

It will turn out that the constants in our error estimates are determined by the interpolation error of the quadratic function $\|\cdot\|^{2}$. We therefore introduce the (nonnegative) functions

$$
e_{y}:=\|\cdot\|^{2}-\Lambda_{y}\left[\|\cdot\|^{2}\right]
$$

where $y \in \mathfrak{P}$, and

$$
e^{\mathrm{v}}:=\Lambda^{\mathrm{v}}\left[\|\cdot\|^{2}\right]-\|\cdot\|^{2}=\sum_{i=1}^{n}\left\|v_{i}\right\|^{2} B_{i}-\|\cdot\|^{2} .
$$

Representations, interrelations, and estimates for these functions are stated in the following lemma.

LEMMA 2.3. The functions $e_{y}$ and $e^{\mathrm{v}}$ are non-negative and vanish at the interpolation points of $\Lambda_{y}$ and $\Lambda^{\mathrm{v}}$, respectively. They satisfy the equations

$$
\begin{aligned}
e_{y} & =\|\cdot-y\|^{2}, \\
e^{\mathrm{v}} & =\sum_{i=1}^{n}\left\|\cdot-v_{i}\right\|^{2} B_{i}, \\
e^{\mathrm{v}}+e_{y} & =\sum_{i=1}^{n} e_{y}\left(v_{i}\right) B_{i} .
\end{aligned}
$$

Furthermore, denoting by

$$
\mathfrak{B}^{\mathrm{sb}}=:\left\{x \in \mathbb{R}^{d}:\left\|x-x^{\mathrm{sb}}\right\| \leq r^{\mathrm{sb}}\right\}
$$


the smallest ball that contains $\mathfrak{P}$, we have

$$
e^{\mathrm{v}}(x) \leq\left(r^{\mathrm{sb}}\right)^{2}-\left\|x-x^{\mathrm{sb}}\right\|^{2} \leq\left(r^{\mathrm{sb}}\right)^{2}
$$

for all $x \in \mathfrak{P}$.

For notational simplicity, we write

$$
\Lambda^{\mathrm{sb}}:=\Lambda_{y} \quad \text { and } \quad e^{\mathrm{sb}}:=e_{y} \quad \text { if } y=x^{\mathrm{sb}} .
$$

Proof. From the definition of the functions $e_{y}$ and $e^{\mathrm{v}}$, it is clear that they vanish at the interpolation points of $\Lambda_{y}$ and $\Lambda^{\mathrm{v}}$, respectively. Since $\|\cdot\|^{2}$ is a convex function, the statements (iii) of Lemmas 2.1 and 2.2 show that $e_{y}$ and $e^{\mathrm{v}}$ are non-negative.

Next, from the definition of $e_{y}$, we deduce that

$$
e_{y}(x)=\|x\|^{2}-\left(\|y\|^{2}+2\langle y, x-y\rangle\right)=\|x\|^{2}+\|y\|^{2}-2\langle y, x\rangle=\|x-y\|^{2},
$$

which is (2.3).

Since $e^{\mathrm{v}}+e_{y}$ belongs to $\mathcal{R}_{n}$, statement (ii) of Lemma 2.2 shows that, for any $x \in \mathfrak{P}$, we have

$$
e^{\mathrm{v}}(x)+e_{y}(x)=\sum_{i=1}^{n}\left(e^{\mathrm{v}}\left(v_{i}\right)+e_{y}\left(v_{i}\right)\right) B_{i}(x)=\sum_{i=1}^{n} e_{y}\left(v_{i}\right) B_{i}(x),
$$

which is (2.5).

Substituting $y=x$ in (2.9) and using (2.3), we obtain (2.4).

For a proof of (2.7), we first note that $x^{\text {sb }} \in \mathfrak{P}$, as a consequence of the convexity of $\mathfrak{P}$. Since

$$
h^{\mathrm{sb}}:=\left(r^{\mathrm{sb}}\right)^{2}-\left\|\cdot-x^{\mathrm{sb}}\right\|^{2}
$$

is non-negative on $\mathfrak{P}$, while $e^{\mathrm{v}}$ vanishes at all the vertices of $\mathfrak{P}$, we clearly have

$$
h^{\mathrm{sb}}\left(v_{i}\right)-e^{\mathrm{v}}\left(v_{i}\right) \geq 0 \quad(i=1, \ldots, n) .
$$

Therefore statement (iv) of Lemma 2.2 implies that $\Lambda^{\mathrm{v}}\left[h^{\mathrm{sb}}-e^{\mathrm{v}}\right] \geq 0$. Furthermore, using (2.3), (2.5), and the notation (2.8), we find that

$$
h^{\mathrm{sb}}-e^{\mathrm{v}}=\left(r^{\mathrm{sb}}\right)^{2}-e^{\mathrm{sb}}-e^{\mathrm{v}}=\left(r^{\mathrm{sb}}\right)^{2}-\sum_{i=1}^{n} e^{\mathrm{sb}}\left(v_{i}\right) B_{i},
$$

which obviously belongs to $\mathcal{R}_{n}$. Hence statement (ii) of Lemma 2.2 allows us to conclude that

$$
h^{\mathrm{sb}}-e^{\mathrm{v}}=\Lambda^{\mathrm{v}}\left[h^{\mathrm{sb}}-e^{\mathrm{v}}\right] \geq 0,
$$

which gives (2.7) immediately.

REMARK 2.4. Inequality (2.7) is of interest for the following reason. As we shall see, the best constants in our error estimates for $\Lambda^{\mathrm{v}}[f]$ are determined by norms of $e^{\mathrm{v}}$. If $e^{\mathrm{v}}$ is complicated, then we may use the simpler function (2.10) instead and obtain a constant which is possibly somewhat worse, but which may still be good enough for practical applications. In the case where $\mathfrak{P}$ is a simplex, it can even be shown that

$$
\sup _{x \in \mathfrak{P}} e^{\mathrm{v}}(x)=\sup _{x \in \mathfrak{P}} h^{\mathrm{sb}}(x)=\left(r^{\mathrm{sb}}\right)^{2} ;
$$

see [6, Lemma 4.2]. 
3. Approximation of Functions. We are mainly interested in approximation of functions from $C^{2}(\mathfrak{P})$. However, in the case where $\mathcal{P}$ is a simplex, Stämpfle $[6$, Theorem 4.1, statements (i)-(iv)] also presented results for functions belonging to lower regularity classes. These statements extend to $\Lambda^{\mathrm{v}}$ by exactly the same arguments as in [6]. We only mention a result for a Lipschitz class which is more general than the one considered in $[6]$.

For $\alpha \in(0,1]$ and $L>0$, we write $f \in \operatorname{Lip}_{L}(\alpha, \mathfrak{P})$ and say that $f$ satisfies a Lipschitz condition of order $\alpha$ with Lipschitz constant $L$ on $\mathfrak{P}$ if $f \in C(\mathfrak{P})$ and

$$
|f(x)-f(y)| \leq L\|x-y\|^{\alpha} \quad(x, y \in \mathfrak{P}) .
$$

Theorem 3.1. Let $f \in \operatorname{Lip}_{L}(\alpha, \mathfrak{P})$. Then

$$
\left|f(x)-\Lambda^{\mathrm{v}}[f](x)\right| \leq L\left(e^{\mathrm{v}}(x)\right)^{\alpha / 2} \quad(x \in \mathfrak{P})
$$

and, for each $p \in[1, \infty]$,

$$
\left\|f-\Lambda^{\mathrm{v}}[f]\right\|_{p} \leq L\left\|\left(e^{\mathrm{v}}\right)^{\alpha / 2}\right\|_{p} .
$$

Proof. From (1.2) and the definition of $\Lambda^{\mathrm{v}}$, it is clear that

$$
f(x)-\Lambda^{\mathrm{v}}[f](x)=\sum_{i=1}^{n}\left(f(x)-f\left(v_{i}\right)\right) B_{i}(x),
$$

and so, by the triangle inequality and the Lipschitz condition,

$$
\left|f(x)-\Lambda^{\mathrm{v}}[f](x)\right| \leq L \sum_{i=1}^{n}\left\|x-v_{i}\right\|^{\alpha} B_{i}(x) .
$$

Next, using Hölder's inequality with $p:=2 / \alpha$ and $q:=2 /(2-\alpha)$, which is an admissible pair of exponents, and recalling (1.2) and (2.4), we find that

$$
\begin{aligned}
\sum_{i=1}^{n}\left\|x-v_{i}\right\|^{\alpha} B_{i}(x) & =\sum_{i=1}^{n}\left\|x-v_{i}\right\|^{\alpha} B_{i}(x)^{1 / p} \cdot B_{i}(x)^{1 / q} \\
& \leq\left(\sum_{i=1}^{n}\left\|x-v_{i}\right\|^{\alpha p} B_{i}(x)\right)^{1 / p} \cdot\left(\sum_{i=1}^{n} B_{i}(x)\right)^{1 / q} \\
& =\left(\sum_{i=1}^{n}\left\|x-v_{i}\right\|^{2} B_{i}(x)\right)^{\alpha / 2}=\left(e^{\mathrm{v}}(x)\right)^{\alpha / 2}
\end{aligned}
$$

Combining this with (3.3), we obtain (3.1). Clearly, (3.2) is an immediate consequence of (3.1).

For twice differentiable functions $f: \mathfrak{P} \rightarrow \mathbb{R}$, we denote by

$$
H[f](x):=\left(\frac{\partial^{2} f}{\partial x_{i} \partial x_{j}}(x)\right)_{i, j=1, \ldots, d}
$$


the Hessian matrix of $f$ at $x$ and introduce

$$
\left|D^{2} f\right|:=\sup _{x \in \mathfrak{P}} \sup _{\substack{y \in \mathbb{R}^{d} \\\|y\|=1}}\left|y^{\top} H[f](x) y\right|,
$$

agreeing that the elements of $\mathbb{R}^{d}$ are column vectors so that $y^{\top}$, which denotes the transpose of $y$, becomes a row vector. Clearly, $\left|D^{2} f\right|=0$ for $f \in \mathcal{P}_{1}$ and $\left|D^{2} f\right|=$ $2|c|$ for $f=c\|\cdot\|^{2}$.

Subsequently, we shall often refer to the space

$$
\mathcal{F}_{2}:=\left\{f:=\lambda+c\|\cdot\|^{2}: \lambda \in \mathcal{P}_{1}, c \in \mathbb{R}\right\}
$$

The following theorem for $\Lambda_{y}$ is not more than an easy exercise in calculus. We formulate it as a theorem only in order to compare it with the corresponding result for $\Lambda^{\mathrm{v}}$.

TheOREM 3.2. Let $f \in C^{2}(\mathfrak{P})$. Then,

$$
\left|f(x)-\Lambda_{y}[f](x)\right| \leq \frac{1}{2}\|x-y\|^{2}\left|D^{2} f\right| \quad(x, y \in \mathfrak{P}) .
$$

Furthermore, for each $p \in[1, \infty]$,

$$
\left\|f-\Lambda_{y}[f]\right\|_{p} \leq c_{y, p}\left|D^{2} f\right|
$$

where

$$
c_{y, p}:=\frac{1}{2}\left\|e_{y}\right\|_{p}
$$

Both inequalities are sharp. Equality is attained for every $f \in \mathcal{F}_{2}$.

Proof. By the Taylor formula of order two, we have

$$
f(x)-\Lambda_{y}[f](x)=\frac{1}{2}(x-y)^{\top} H[f](y+\theta(x-y))(x-y)
$$

for some $\theta \in(0,1)$. Now the definition of $\left|D^{2} f\right|$, given in (3.4), shows that (3.6) holds. Inequality (3.7) is an immediate consequence of (3.6). Finally, the case of equality is easily verified.

TheOREM 3.3. Let $f \in C^{2}(\mathfrak{P})$. Then,

$$
\left|f(x)-\Lambda^{\mathrm{v}}[f](x)\right| \leq \frac{1}{2} e^{\mathrm{v}}(x)\left|D^{2} f\right| \quad(x \in \mathfrak{P}) .
$$

Furthermore, for each $p \in[1, \infty]$,

$$
\left\|f-\Lambda^{\mathrm{v}}[f]\right\|_{p} \leq c_{p}^{\mathrm{v}}\left|D^{2} f\right|
$$

where

$$
c_{p}^{\mathrm{v}}:=\frac{1}{2}\left\|e^{\mathrm{v}}\right\|_{p}
$$

Both inequalities are sharp. Equality is attained for every $f \in \mathcal{F}_{2}$. 
Proof. Inequality (3.6) may be rewritten as

$$
-\frac{1}{2}\|x-y\|^{2}\left|D^{2} f\right| \leq f(x)-\Lambda_{y}[f](x) \leq \frac{1}{2}\|x-y\|^{2}\left|D^{2} f\right| \quad(x, y \in \mathfrak{P}) .
$$

Next, from statement (iv) of Lemma 2.2, it follows that inequalities between continuous functions on $\mathfrak{P}$ are preserved when the operator $\Lambda^{\mathrm{v}}$ is applied on both sides. Moreover, statement (i) of Lemma 2.1 together with statement (ii) of Lemma 2.2 show that

$$
\Lambda^{\mathrm{v}}\left[\Lambda_{y}[f]\right]=\Lambda_{y}[f]
$$

Hence (3.12) implies that

$$
-\frac{1}{2} \Lambda^{\mathrm{v}}\left[\|\cdot-y\|^{2}\right](x)\left|D^{2} f\right| \leq \Lambda^{\mathrm{v}}[f](x)-\Lambda_{y}[f](x) \leq \frac{1}{2} \Lambda^{\mathrm{v}}\left[\|\cdot-y\|^{2}\right](x)\left|D^{2} f\right| .
$$

Now, taking $y=x$ and noting that $\Lambda_{x}[f](x)=f(x)$ and, by $(2.4)$,

$$
\Lambda^{\mathrm{v}}\left[\|\cdot-x\|^{2}\right](x)=\sum_{i=1}^{n}\left\|v_{i}-x\right\|^{2} B_{i}(x)=e^{\mathrm{v}}(x),
$$

we obtain

$$
-\frac{1}{2} e^{\mathrm{v}}(x)\left|D^{2} f\right| \leq \Lambda^{\mathrm{v}}[f](x)-f(x) \leq \frac{1}{2} e^{\mathrm{v}}(x)\left|D^{2} f\right|
$$

which is equivalent to (3.9). Inequality (3.10) is an immediate consequence of (3.9). The statement on the occurrence of equality is easily verified by a calculation.

REMARK 3.4. Since $\Lambda^{\mathrm{v}}$ is a positive operator which reproduces affine functions, inequality (3.9) can also be deduced from [9, Theorem 1.4] in conjunction with the above Lemma 2.3 .

The operator $\Lambda_{y}$ has just one interpolation point, which is of multiplicity two. Such an interpolation can be described by $d+1$ scalar equations. The interpolation of the operator $\Lambda^{\mathrm{v}}$, which has $n$ simple interpolation points, can be described by $n$ scalar equations. Since $n \geq d+1$, we may expect that the operator $\Lambda^{\mathrm{v}}$ is at least as precise as $\Lambda_{y}$. In the following proposition, we compare the constants (3.8) and (3.11) when $p=\infty$.

Proposition 3.5. For $p=\infty$, the constants (3.8) and (3.11) satisfy the relations

$$
c_{\infty}^{\mathrm{v}} \leq c_{y, \infty} \quad(y \in \mathfrak{P})
$$

and

$$
\inf _{y \in \mathfrak{P}} c_{y, \infty}=\frac{\left(r^{\mathrm{sb}}\right)^{2}}{2}
$$

the infimum being attained for $y=x^{\mathrm{sb}}$, where $r^{\mathrm{sb}}$ and $x^{\mathrm{sb}}$ specify the smallest ball $\mathfrak{B}^{\text {sb }}$ which contains $\mathfrak{P}$, as introduced in (2.6).

If all the vertices of $\mathfrak{P}$ lie on the boundary of $\mathfrak{B}^{\mathrm{sb}}$, then

$$
c_{\infty}^{\mathrm{v}}=\frac{\left(r^{\mathrm{sb}}\right)^{2}}{2}
$$


Proof. It follows from (2.5) that

$$
e^{\mathrm{v}}(x) \leq \sum_{i=1}^{n} e_{y}\left(v_{i}\right) B_{i}(x) \leq \max _{1 \leq i \leq n} e_{y}\left(v_{i}\right) \quad(x \in \mathfrak{P}),
$$

which implies (3.13).

Since a convex function, defined on a convex set, attains its supremum at an extreme point (see for example [4, p. 91]), we have

$$
\max _{1 \leq i \leq n} e_{y}\left(v_{i}\right)=\sup _{x \in \mathfrak{P}} e_{y}(x)=2 c_{y, \infty} .
$$

This shows that $c_{y, \infty}$ attains its smallest value at a point where

$$
\phi(y):=\max _{1 \leq i \leq n} e_{y}\left(v_{i}\right)=\max _{1 \leq i \leq n}\left\|y-v_{i}\right\|^{2}
$$

attains its minimum. Clearly, this is the center of the smallest ball $\mathfrak{B}^{\text {sb }}$ that contains $\mathfrak{P}$, and so

$$
\min _{y \in \mathfrak{P}} \phi(y)=\phi\left(x^{\mathrm{sb}}\right)=\left(r^{\mathrm{sb}}\right)^{2}
$$

Thus (3.14) is verified.

If all the vertices of $\mathfrak{P}$ lie on the boundary of $\mathfrak{B}^{\mathrm{sb}}$, then $\left\|x^{\mathrm{sb}}-v_{i}\right\|=r^{\mathrm{sb}}$ for $i=1, \ldots, n$. Therefore, by $(2.4)$,

$$
e^{\mathrm{v}}\left(x^{\mathrm{sb}}\right)=\sum_{i=1}^{n}\left\|x^{\mathrm{sb}}-v_{i}\right\|^{2} B_{i}\left(x^{\mathrm{sb}}\right)=\left(r^{\mathrm{sb}}\right)^{2} \sum_{i=1}^{n} B_{i}\left(x^{\mathrm{sb}}\right)=\left(r^{\mathrm{sb}}\right)^{2},
$$

which shows that $c_{\infty}^{\mathrm{v}} \geq\left(r^{\mathrm{sb}}\right)^{2} / 2$. Combining this inequality with (3.13) and (3.14), we obtain (3.15).

In the univariate case, where $\mathfrak{P}$ is an interval $[a, b]$, it is known and also seen from (3.15) that, for $y=(b+a) / 2$, we have

$$
c_{\infty}^{\mathrm{v}}=c_{y, \infty}=\frac{(b-a)^{2}}{8}
$$

Moreover, the mean value

$$
\frac{1}{2}\left(\Lambda_{y}[f]+\Lambda^{\mathrm{v}}[f]\right) \quad\left(y=\frac{a+b}{2}\right)
$$

gives an approximation whose constant in the error bound is $(b-a)^{2} / 16$. A generalization is given in the following proposition.

Proposition 3.6. Let $f \in C^{2}(\mathfrak{P})$. Then, for every $y \in \mathfrak{P}$ and $\alpha \in[0,1]$, we have

$$
\left\|f-\alpha \Lambda_{y}[f]-(1-\alpha) \Lambda^{\mathrm{v}}[f]\right\|_{\infty} \leq c(\alpha, y)\left|D^{2} f\right|
$$

where

$$
c(\alpha, y):=\frac{1}{2} \sup _{x \in \mathfrak{P}}\left(\alpha e_{y}(x)+(1-\alpha) e^{\mathrm{v}}(x)\right) .
$$


Furthermore,

$$
\inf _{0 \leq \alpha \leq 1} \inf _{y \in \mathfrak{P}} c(\alpha, y) \leq \frac{\left(r^{\mathrm{sb}}\right)^{2}}{4}=c\left(\frac{1}{2}, x^{\mathrm{sb}}\right)
$$

where $r^{\mathrm{sb}}$ and $x^{\mathrm{sb}}$ are the radius and the center of the smallest ball $\mathfrak{B}^{\mathrm{sb}}$ that contains $\mathfrak{P}$. Equality occurs in (3.20) if all the vertices of $\mathfrak{P}$ lie on the boundary of $\mathfrak{B}^{\text {sb }}$. In this case, inequality (3.18) is sharp when $\alpha=1 / 2$ and $y=x^{\mathrm{sb}}$, and equality is attained for every function $f \in \mathcal{F}_{2}$.

Proof. The estimates (3.6) and (3.9) may be rewritten as

$$
-\frac{1}{2} e_{y}(x)\left|D^{2} f\right| \leq f(x)-\Lambda_{y}[f](x) \leq \frac{1}{2} e_{y}(x)\left|D^{2} f\right|
$$

and

$$
-\frac{1}{2} e^{\mathrm{v}}(x)\left|D^{2} f\right| \leq f(x)-\Lambda^{\mathrm{v}}[f](x) \leq \frac{1}{2} e^{\mathrm{v}}(x)\left|D^{2} f\right|
$$

Multiplying the first inequalities by $\alpha$ and the second by $1-\alpha$, and adding the results, we obtain

$$
\left|f(x)-\alpha \Lambda_{y}[f](x)-(1-\alpha) \Lambda^{\mathrm{v}}[f](x)\right| \leq \frac{1}{2}\left(\alpha e_{y}(x)+(1-\alpha) e^{\mathrm{v}}(x)\right)\left|D^{2} f\right| .
$$

This implies (3.18).

Next, using (2.5) and the notation (2.8), we find that

$$
c\left(\frac{1}{2}, x^{\mathrm{sb}}\right)=\frac{1}{4} \sup _{x \in \mathfrak{P}}\left(e^{\mathrm{v}}(x)+e^{\mathrm{sb}}(x)\right)=\frac{1}{4} \sup _{x \in \mathfrak{P}} \sum_{i=1}^{n}\left\|x^{\mathrm{sb}}-v_{i}\right\|^{2} B_{i}(x) .
$$

If $v_{j}$ is a vertex on the boundary of $\mathfrak{B}^{\text {sb }}$, then, by (1.1), (1.3), (2.11), and (2.12),

$$
\sum_{i=1}^{n}\left\|x^{\mathrm{sb}}-v_{i}\right\|^{2} B_{i}\left(v_{j}\right)=\left\|x^{\mathrm{sb}}-v_{j}\right\|^{2}=\left(r^{\mathrm{sb}}\right)^{2} \geq \sum_{i=1}^{n}\left\|x^{\mathrm{sb}}-v_{i}\right\|^{2} B_{i}(x)
$$

for all $x \in \mathfrak{P}$. This shows that

$$
\sup _{x \in \mathfrak{P}} \sum_{i=1}^{n}\left\|x^{\mathrm{sb}}-v_{i}\right\|^{2} B_{i}(x)=\left(r^{\mathrm{sb}}\right)^{2}
$$

and completes the proof of (3.20).

Using (3.19), we deduce that

$$
c(\alpha, y) \geq \frac{1-\alpha}{2} \sup _{x \in \mathfrak{P}} e^{\mathrm{v}}(x)=(1-\alpha) c_{\infty}^{\mathrm{v}} \geq \frac{c_{\infty}^{\mathrm{v}}}{2} \quad \text { if } \alpha \in\left[0, \frac{1}{2}\right]
$$

and, in conjunction with (3.14),

$$
c(\alpha, y) \geq \frac{\alpha}{2} \sup _{x \in \mathfrak{P}} e_{y}(x)=\alpha c_{y, \infty} \geq \frac{\left(r^{\mathrm{sb}}\right)^{2}}{4} \quad \text { if } \alpha \in\left[\frac{1}{2}, 1\right] .
$$


Under the hypothesis that all the vertices of $\mathfrak{P}$ lie on the boundary of $\mathfrak{B}^{\text {sb }}$, we know from Proposition 3.5 that

$$
c_{\infty}^{\mathrm{v}}=\frac{\left(r^{\mathrm{sb}}\right)^{2}}{2}
$$

Hence

$$
c(\alpha, y) \geq \frac{\left(r^{\mathrm{sb}}\right)^{2}}{4} \quad(\alpha \in[0,1], y \in \mathfrak{P})
$$

which shows that equality occurs in (3.20).

Finally, we have to verify the statement on the occurrence of equality for functions $f$ from the class $\mathcal{F}_{2}$. For this, it is clearly enough to consider the function $f:=\|\cdot\|^{2}$ only.

Using the notation (2.8), we may rewrite (2.1) and (2.2) as

$$
\begin{aligned}
f(x)-\Lambda^{\mathrm{sb}}[f](x) & =e^{\mathrm{sb}}(x), \\
f(x)-\Lambda^{\mathrm{v}}[f](x) & =-e^{\mathrm{v}}(x) .
\end{aligned}
$$

Therefore,

$$
f(x)-\frac{1}{2} \Lambda^{\mathrm{sb}}[f](x)-\frac{1}{2} \Lambda^{\mathrm{v}}[f](x)=\frac{1}{2}\left(e^{\mathrm{sb}}(x)-e^{\mathrm{v}}(x)\right)
$$

and consequently,

$$
\left\|f-\frac{1}{2} \Lambda^{\mathrm{sb}}[f]-\frac{1}{2} \Lambda^{\mathrm{v}}[f]\right\|_{\infty}=\frac{1}{2} \sup _{x \in \mathfrak{P}}\left|e^{\mathrm{sb}}(x)-e^{\mathrm{v}}(x)\right| .
$$

If all the vertices of $\mathfrak{P}$ lie on the boundary of $\mathfrak{B}^{\text {sb }}$, then

$$
\sup _{x \in \mathfrak{P}}\left|e^{\mathrm{sb}}(x)-e^{\mathrm{v}}(x)\right| \geq\left|e^{\mathrm{sb}}\left(x^{\mathrm{sb}}\right)-e^{\mathrm{v}}\left(x^{\mathrm{sb}}\right)\right|=e^{\mathrm{v}}\left(x^{\mathrm{sb}}\right)=\left(r^{\mathrm{sb}}\right)^{2},
$$

where the last equation follows from (2.4) and (1.2), and so

$$
\left\|f-\frac{1}{2} \Lambda^{\mathrm{sb}}[f]-\frac{1}{2} \Lambda^{\mathrm{v}}[f]\right\|_{\infty} \geq \frac{\left(r^{\mathrm{sb}}\right)^{2}}{2} .
$$

On the other hand, (3.18) and (3.20) show that

$$
\left\|f-\frac{1}{2} \Lambda^{\mathrm{sb}}[f]-\frac{1}{2} \Lambda^{\mathrm{v}}[f]\right\|_{\infty} \leq \frac{\left(r^{\mathrm{sb}}\right)^{2}}{2} .
$$

Hence equality occurs for $f=\|\cdot\|^{2}$.

4. Approximation of Linear Functionals. In the case $p=1$, Theorems 3.13.3 provide an approximation of $\mathcal{L}(f)$, defined in (1.4), by the values of $f$ (and possibly of $D f)$ at the interpolation points of $\Lambda_{y}$ and $\Lambda^{\mathrm{v}}$, respectively. Indeed, if $\Lambda$ is any of the two operators $\Lambda_{y}$ and $\Lambda^{\mathrm{v}}$, and $I(f):=\mathcal{L}(\Lambda[f])$, then, using that $\mathcal{L}$ is linear and positive, we have

$$
|\mathcal{L}(f)-I(f)|=|\mathcal{L}(f-\Lambda[f])| \leq \mathcal{L}(|f-\Lambda[f]|)=\|f-\Lambda[f]\|_{1} .
$$


Let us now turn to details. Denoting by id the identity mapping on $\mathfrak{P}$ and observing that $\mathcal{L}(\mathrm{id})$ is a mapping from $\mathfrak{P}$ into $\mathbb{R}^{d}$, we shall consider the operators

$$
I_{y}(f):=\mathcal{L}\left(\Lambda_{y}[f]\right)=\mathcal{L}(1)\left[f(y)+D f(y)\left(\frac{\mathcal{L}(\mathrm{id})}{\mathcal{L}(1)}-y\right)\right]
$$

and

$$
I^{\mathrm{v}}(f):=\mathcal{L}\left(\Lambda^{\mathrm{v}}[f]\right)=\sum_{i=1}^{n} f\left(v_{i}\right) \mathcal{L}\left(B_{i}\right) .
$$

In the case $p=1$, the constants (3.8) and (3.11) can be expressed as

$$
c_{y, 1}=\frac{1}{2} \mathcal{L}\left(e_{y}\right) \quad \text { and } \quad c_{1}^{\mathrm{v}}=\frac{1}{2}\left(I^{\mathrm{v}}\left(e_{y}\right)-\mathcal{L}\left(e_{y}\right)\right) .
$$

Note that the last equation, which is deduced with the help of (2.5), is independent of $y$. Now Theorems 3.2 and 3.3 imply the following corollaries.

Corollary 4.1. Let $f \in C^{2}(\mathfrak{P})$. Then, for any $y \in \mathfrak{P}$, we have

$$
\left|\mathcal{L}(f)-I_{y}(f)\right| \leq \frac{\mathcal{L}\left(e_{y}\right)}{2}\left|D^{2} f\right| .
$$

Equality is attained for every $f \in \mathcal{F}_{2}$.

Corollary 4.2. Let $f \in C^{2}(\mathfrak{P})$. Then, for any $y \in \mathfrak{P}$, we have

$$
\left|\mathcal{L}(f)-I^{\mathrm{v}}(f)\right| \leq \frac{I^{\mathrm{v}}\left(e_{y}\right)-\mathcal{L}\left(e_{y}\right)}{2}\left|D^{2} f\right| .
$$

Equality is attained for every $f \in \mathcal{F}_{2}$.

Remark 4.3. The conclusions of Corollaries 4.1 and 4.2 can be refined when, in addition, $f$ is known to be a convex function. In fact, in this case, we also have

$$
I_{y}(f) \leq \mathcal{L}(f) \leq I^{\mathrm{v}}(f)
$$

as a consequence of the statements (iii) of Lemmas 2.1 and 2.2.

The "cubature rule" $I^{\mathrm{v}}(f)$ may be seen as a multivariate analogue of the trapezoidal rule. As (4.1) shows, the "cubature rule" $I_{y}(f)$ simplifies and does not depend on $D f$ if $y$ is chosen as

$$
x^{\mathrm{cm}}:=\frac{\mathcal{L}(\mathrm{id})}{\mathcal{L}(1)} .
$$

In this case, $I_{y}(f)$ is a multivariate analogue of the midpoint rule.

The point $x^{\mathrm{cm}}$ will be called the center of mass of $\mathfrak{P}$ with respect to the functional $\mathcal{L}$. Note that $x^{\mathrm{cm}}$ always belongs to $\mathfrak{P}$. Indeed, if $x^{\mathrm{cm}}$ were outside $\mathfrak{P}$, then there would exist a separating hyperplane

$$
\lambda(x):=a+\langle b, x\rangle=0,
$$

where $a \in \mathbb{R}$ and $b \in \mathbb{R}^{d}$, such that $\lambda(x)>0$ for $x \in \mathfrak{P}$ and $\lambda\left(x^{\mathrm{cm}}\right)<0$. Since $\mathcal{L}$ is positive, we would have $\mathcal{L}(\lambda)>0$. On the other hand, the linearity of $\mathcal{L}$ implies that

$$
\mathcal{L}(\lambda)=a \mathcal{L}(1)+\langle b, \mathcal{L}(\mathrm{id})\rangle=a \mathcal{L}(1)+\left\langle b, \mathcal{L}(1) x^{\mathrm{cm}}\right\rangle=\mathcal{L}(1) \lambda\left(x^{\mathrm{cm}}\right)<0,
$$


which is a contradiction.

For notational simplicity, we now write

$$
\Lambda^{\mathrm{cm}}:=\Lambda_{y}, \quad I^{\mathrm{cm}}:=I_{y}, \quad e^{\mathrm{cm}}:=e_{y}, \quad c_{p}^{\mathrm{cm}}:=c_{y, p} \quad \text { if } y=x^{\mathrm{cm}} .
$$

Since

$$
e_{y}(x)=\|x-y\|^{2}=\left\|x-x^{\mathrm{cm}}\right\|^{2}+\left\|x^{\mathrm{cm}}-y\right\|^{2}+2\left\langle x-x^{\mathrm{cm}}, x^{\mathrm{cm}}-y\right\rangle,
$$

we find, using the definition of $x^{\mathrm{cm}}$, that

$$
c_{y, 1}=\mathcal{L}\left(e_{y}\right)=\mathcal{L}\left(e^{\mathrm{cm}}\right)+\mathcal{L}(1)\left\|x^{\mathrm{cm}}-y\right\|^{2} .
$$

This shows that the constant in the error estimate of Corollary 4.1 becomes smallest if and only if $y=x^{\mathrm{cm}}$.

REMARK 4.4. It may be interesting to compare the operators $I^{\mathrm{cm}}$ and $I^{\mathrm{v}}$. Recalling that $c_{1}^{\mathrm{v}}$ in (4.3) does not depend on $y$, we may take $y=x^{\mathrm{cm}}$. Then Corollaries 4.1 and 4.2 show that the quotient

$$
\kappa:=\frac{\mathcal{L}\left(e^{\mathrm{cm}}\right)}{I^{\mathrm{v}}\left(e^{\mathrm{cm}}\right)}
$$

indicates which one of the two operators $I^{\mathrm{cm}}$ and $I^{\mathrm{v}}$ has the smaller constant in its error estimate. We see that $c_{1}^{\mathrm{cm}}<c_{1}^{\mathrm{v}}$ if and only if $\kappa \in(0,1 / 2)$. Since, for convex functions, $I^{\mathrm{v}}$ approximates $\mathcal{L}$ from above, we always have $\kappa \in(0,1)$. In all the standard examples considered by us, we found that $\kappa \in(0,1 / 2)$. However, $\kappa \in[1 / 2,1)$ will occur when $\mathcal{L}$ is of the form (1.4) and the weight function $w$ is large near the vertices.

5. Examples. We illustrate our results by considering three special classes of convex polytopes for which interpolation and approximation problems have been studied in the literature.

5.1. Intervals (the univariate case). Let $d:=1, \mathfrak{P}:=[a, b]$, and $\mathcal{L}(f):=$ $\int_{a}^{b} f(x) \mathrm{d} x$. Then $x^{\mathrm{sb}}=x^{\mathrm{cm}}=\frac{1}{2}(a+b)$,

$$
\Lambda^{\mathrm{cm}}[f](x)=f\left(\frac{a+b}{2}\right)+f^{\prime}\left(\frac{a+b}{2}\right)\left(x-\frac{a+b}{2}\right)
$$

and

$$
\Lambda^{\mathrm{v}}[f](x)=\frac{b-x}{b-a} f(a)+\frac{x-a}{b-a} f(b) .
$$

Moreover, $\left|D^{2} f\right|=\sup _{a \leq x \leq b}\left|f^{\prime \prime}(x)\right|$. For the constants (3.8) with $y=x^{\mathrm{cm}}$ and (3.11), we find that

$$
c_{p}^{\mathrm{cm}}=\frac{1}{2}\left[\frac{(b-a)^{2 p+1}}{2^{2 p}(2 p+1)}\right]^{1 / p} \quad(1 \leq p<\infty)
$$

and

$$
c_{p}^{\mathrm{v}}=\frac{1}{2}\left[B(p+1, p+1)(b-a)^{2 p+1}\right]^{1 / p} \quad(1 \leq p<\infty)
$$


where

$$
B(s, t):=\int_{0}^{1} x^{s-1}(1-x)^{t-1} \mathrm{~d} x
$$

is the beta function. Furthermore,

$$
c_{\infty}^{\mathrm{cm}}=c_{\infty}^{\mathrm{v}}=\frac{(b-a)^{2}}{8} .
$$

It can be shown that $c_{p}^{\mathrm{cm}}<c_{p}^{\mathrm{v}}$ for $1 \leq p<\infty$. In particular, $c_{1}^{\mathrm{v}} / c_{1}^{\mathrm{cm}}=2$, which expresses the well known fact that the constant in the error term of the trapezoidal rule is twice as large as that of the midpoint rule.

5.2. Multidimensional simplices. Let $\mathfrak{S} \subset \mathbb{R}^{d}$ be a non-degenerate simplex with vertices $v_{0}, \ldots, v_{d}$. The uniquely determined rational basis functions $B_{0}, \ldots, B_{d}$ of minimal degree are the classical barycentric coordinates, which may be constructed as follows. Let $\lambda_{i}(x)=0$ be the equation of a hyperplane that contains all the vertices of $\mathfrak{S}$ other than $v_{i}$. Then

$$
B_{i}(x)=\frac{\lambda_{i}(x)}{\lambda_{i}\left(v_{i}\right)} \quad(i=0, \ldots, d) .
$$

For $\mathcal{L}(f):=\int_{\mathfrak{S}} f(x) \mathrm{d} x$, we obtain

$$
x^{\mathrm{cm}}=\frac{1}{|\mathfrak{S}|} \int_{\mathfrak{S}} x \mathrm{~d} x=\frac{1}{d+1} \sum_{i=0}^{d} v_{i},
$$

where we write $|\mathfrak{S}|$ for the $d$-dimensional volume of $\mathfrak{S}$. This gives a representation of $e^{\mathrm{cm}}$ in terms of the vertices, which, via (4.4) and (3.17), leads us to

$$
c_{\infty}^{\mathrm{cm}}=\frac{1}{2(d+1)^{2}} \max _{0 \leq i \leq d}\left\|\sum_{j=0}^{d}\left(v_{i}-v_{j}\right)\right\|^{2} .
$$

Since the basis functions $B_{i}$ belong to $\mathcal{P}_{1}$, the function $e^{\mathrm{v}}$, defined in $(2.2)$, is now of the form $e^{\mathrm{v}}=\lambda-\|\cdot\|^{2}$, where $\lambda \in \mathcal{P}_{1}$. Therefore $e^{\mathrm{v}}(x)=0$ is the equation of the uniquely defined sphere that contains all the vertices of $\mathfrak{S}$ (see e.g., Stämpfle $[6$, Proposition 3.1]). Thus $e^{\mathrm{v}}$ can be represented as

$$
e^{\mathrm{v}}(x)=\widehat{r}^{2}-\|x-\widehat{x}\|^{2}
$$

for some $\widehat{r}>0$ and $\widehat{x} \in \mathbb{R}^{d}$.

The case of the approximation by $\Lambda^{\mathrm{v}}$ with respect to the norm $\|\cdot\|_{\infty}$ is covered by the papers of Waldron [8, Theorem 2.1] and Stämpfle [6, Theorem 4.1]; also see de Boor [1]. Clearly, $c_{\infty}^{\mathrm{v}}=\widehat{r}^{2} / 2$ when $\widehat{x} \in \mathfrak{S}$. Otherwise, it can be shown that $c_{\infty}^{\mathrm{v}}=\frac{1}{2}\left(\widehat{r}^{2}-\rho^{2}\right)$, where $\rho$ is the distance of $\widehat{x}$ from $\mathfrak{S}$. Geometrically, $2 c_{\infty}^{\mathrm{v}}$ may be interpreted as the square of the radius of the smallest ball that contains $\mathfrak{S}$ (see $[6$, Lemma 4.2]).

For the standard unit simplex of dimension $d \geq 2$, a straightforward calculation gives

$$
c_{\infty}^{\mathrm{cm}}=\frac{d^{2}+d-1}{2(d+1)^{2}} \quad \text { and } \quad c_{\infty}^{\mathrm{v}}=\frac{d-1}{2 d}
$$


and so

$$
\frac{c_{\infty}^{\mathrm{v}}}{c_{\infty}^{\mathrm{cm}}}=1-\frac{1}{d\left(d^{2}+d-1\right)}<1 .
$$

For the calculation of the constants (4.3) for $y=x^{\mathrm{cm}}$, we first determine $\mathcal{L}\left(e^{\mathrm{cm}}\right)$ with the help of a cubature rule which is exact for all polynomials of degree less or equal to two, taken from the book of Stroud [7, p. 307, formula $\left.T_{n}: 2-2\right]$. It gives

$$
\begin{aligned}
\mathcal{L}\left(e^{\mathrm{cm}}\right)=\int_{\mathfrak{S}} e^{\mathrm{cm}}(x) \mathrm{d} x= & \frac{(2-d)|\mathfrak{S}|}{(d+2)(d+1)} \sum_{i=0}^{d} e^{\mathrm{cm}}\left(v_{i}\right) \\
& +\frac{4|\mathfrak{S}|}{(d+2)(d+1)} \sum_{0 \leq i<j \leq d} e^{\mathrm{cm}}\left(v_{i j}\right),
\end{aligned}
$$

where $v_{i j}=\frac{1}{2}\left(v_{i}+v_{j}\right)$. Simplifying the second sum by making use of the special form of $e^{\mathrm{cm}}$, we arrive at

$$
\mathcal{L}\left(e^{\mathrm{cm}}\right)=\frac{|\mathfrak{S}|}{(d+2)(d+1)} \sum_{i=0}^{d} e^{\mathrm{cm}}\left(v_{i}\right)
$$

Since the basis functions $B_{0}, \ldots, B_{d}$ belong to $\mathcal{P}_{1}$, we conclude that

$$
\mathcal{L}\left(B_{i}\right)=\mathcal{L}(1) B_{i}\left(x^{\mathrm{cm}}\right)=\frac{\mathcal{L}(1)}{d+1}=\frac{|\mathfrak{S}|}{d+1} \quad(i=0, \ldots, d)
$$

and therefore

$$
I^{\mathrm{v}}\left(e^{\mathrm{cm}}\right)=\frac{|\mathfrak{S}|}{d+1} \sum_{i=0}^{d} e^{\mathrm{cm}}\left(v_{i}\right) .
$$

Thus, by (4.3), the definition of $e^{\mathrm{cm}}$ in (4.4), and the representation in (2.3), we have

$$
c_{1}^{\mathrm{cm}}=\frac{|\mathfrak{S}|}{2(d+2)(d+1)} \sum_{i=0}^{d}\left\|v_{i}-x^{\mathrm{cm}}\right\|^{2}
$$

and

$$
c_{1}^{\mathrm{v}}=\frac{|\mathfrak{S}|}{2(d+2)} \sum_{i=0}^{d}\left\|v_{i}-x^{\mathrm{cm}}\right\|^{2} .
$$

These values for $c_{1}^{\mathrm{cm}}$ and $c_{1}^{\mathrm{v}}$ also follow from [3, Corollary 6.2, formulae (6.4) and (6.5)]. We see that $c_{1}^{\mathrm{v}}=(d+1) c_{1}^{\mathrm{cm}}$ and $\kappa=1 /(d+2)$ in $(4.5)$.

5.3. Hyperrectangles. Let

$$
\mathfrak{R}:=\left[a_{1}, b_{1}\right] \times \cdots \times\left[a_{d}, b_{d}\right]
$$

be a rectangle in $\mathbb{R}^{d}$ with vertices

$$
v_{i}:=\left(v_{i 1}, \ldots, v_{i d}\right) \quad\left(i=1, \ldots, 2^{d}\right) .
$$

To each vertex $v_{i}$, there exists exactly one vertex of maximal distance, which we call the diametrically opposite vertex and which we denote by $\bar{v}_{i}:=\left(\bar{v}_{i 1}, \ldots, \bar{v}_{i d}\right)$. Any 
two vertices $v_{i}$ and $v_{j}$ have at least one common component unless they are a pair of diametrically opposite vertices. Therefore

$$
B_{i}(x):=\prod_{j=1}^{d} \frac{x_{j}-\bar{v}_{i j}}{v_{i j}-\bar{v}_{i j}} \quad\left(i=1, \ldots, 2^{d}\right),
$$

where $x=\left(x_{1}, \ldots, x_{d}\right)$, are the rational basis functions of smallest degree. They span a polynomial space of dimension $2^{d}$, which contains $\mathcal{P}_{1}$ as a subspace.

For $\mathcal{L}(f):=\int_{\mathfrak{R}} f(x) \mathrm{d} x$, the center of mass is

$$
x^{\mathrm{cm}}=\frac{1}{2}\left(a_{1}+b_{1}, \ldots, a_{d}+b_{d}\right) .
$$

With this, we find that

$$
e^{\mathrm{cm}}\left(v_{i}\right)=\frac{1}{4} \sum_{i=1}^{d}\left(a_{i}-b_{i}\right)^{2}=:\left(r^{\mathrm{cm}}\right)^{2} \quad\left(i=1, \ldots, 2^{d}\right) .
$$

Therefore (2.5) implies that

$$
e^{\mathrm{cm}}(x)+e^{\mathrm{v}}(x)=\left(r^{\mathrm{cm}}\right)^{2}
$$

for all $x$. Consequently,

$$
\sup _{x \in \mathfrak{R}} e^{\mathrm{cm}}(x)=\sup _{x \in \mathfrak{R}} e^{\mathrm{v}}(x)=\left(r^{\mathrm{cm}}\right)^{2},
$$

or equivalently,

$$
c_{\infty}^{\mathrm{cm}}=c_{\infty}^{\mathrm{v}}=\frac{\left(r^{\mathrm{cm}}\right)^{2}}{2} .
$$

For determining the best constants in the case $p=1$, we first calculate

$$
\mathcal{L}\left(e^{\mathrm{cm}}\right)=\int_{\mathfrak{R}}\left\|x-x^{\mathrm{cm}}\right\|^{2} \mathrm{~d} x=\frac{\left(r^{\mathrm{cm}}\right)^{2}}{3}|\mathfrak{R}|,
$$

where $|\mathfrak{R}|=\prod_{i=1}^{d}\left(b_{i}-a_{i}\right)$, and note that

$$
I^{\mathrm{v}}\left(e^{\mathrm{cm}}\right)=\left(r^{\mathrm{cm}}\right)^{2}|\mathfrak{R}| .
$$

Hence (4.3) with $y=x^{\mathrm{cm}}$ implies that

$$
c_{1}^{\mathrm{cm}}=\frac{\left(r^{\mathrm{cm}}\right)^{2}}{6}|\mathfrak{R}| \quad \text { and } \quad c_{1}^{\mathrm{v}}=\frac{\left(r^{\mathrm{cm}}\right)^{2}}{3}|\mathfrak{R}| .
$$

Thus, $c_{1}^{\mathrm{v}} / c_{1}^{\mathrm{cm}}=2$ and $\kappa=1 / 3$, as in the univariate case.

In the literature, analogues of the trapezoidal rule for hyperrectangles have been studied in the context of tensor product rules (see, e.g., $[2, \S 8.2]$ ). 


\section{REFERENCES}

[1] C. DE BooR, Error in linear interpolation at the vertices of a simplex, WWW (1998), http://www.cs.wisc.edu/ deboor/multiint/.

[2] F.-J. Delvos And W. Schempp, Boolean Methods in Interpolation and Approximation, Longman Scientific \& Technical, Essex, 1989.

[3] A. Guessab And G. SChmeisser, Convexity results and sharp error estimates in approximate multivariate integration, Math. Comp., 73 (2004), pp. 1365-1384.

[4] G. Hadley, Nonlinear and Dynamic Programming, Addison-Wesley, Reading, Massachusetts, 1964.

[5] A. W. Roberts and D. E. Varnberg, Convex Sets, Academic Press, New York, 1973.

[6] M. Stämpfle, Optimal estimates for the linear interpolation error on simplices, J. Approx. Theory, 103 (2000), pp. 78-90.

[7] A. H. Stroud, Approximate Calculation of Multiple Integrals, Prentice-Hall, Englewood Cliffs, N. J., 1971.

[8] S. WALDRON, The error in linear interpolation at the vertices of a simplex, SIAM J. Numer. Anal. 35 (1998), pp. 1191-1200.

[9] S. WALDRON, Sharp error estimates for multivariate positive linear operators, in Approximation Theory IX, Volume I, Proceedings of the 9th international conference, Nashville, TN, USA, 1998, C. K. Chui et al., eds., Vanderbilt University Press, Nashville, TN, 1998, pp. 339-346.

[10] J. WARREN, Barycentric coordinates for convex polytopes, Adv. Comput. Math. 6 (1996), pp. 97-108. 